\title{
Numerical Renormalization Group for Impurity Quantum Phase Transitions: Structure of Critical Fixed Points
}

\author{
Hyun-Jung Lee*, Ralf Bulla*, and Matthias Vojta ${ }^{\dagger}$ \\ * Theoretische Physik III, Elektronische Korrelationen und Magnetismus, \\ Institut für Physik, Universität Augsburg, D-86135 Augsburg, Germany and \\ ${ }^{\dagger}$ Institut für Theorie der Kondensierten Materie, \\ Universität Karlsruhe, D-76128 Karlsruhe, Germany
}

(Dated: September 23, 2018)

\begin{abstract}
The numerical renormalization group method is used to investigate zero temperature phase transitions in quantum impurity systems, in particular in the particle-hole symmetric soft-gap Anderson model. The model displays two stable phases whose fixed points can be built up of non-interacting single-particle states. In contrast, the quantum phase transitions turn out to be described by interacting fixed points, and their excitations cannot be described in terms of free particles. We show that the structure of the many-body spectrum of these critical fixed points can be understood using renormalized perturbation theory close to certain values of the bath exponents which play the role of critical dimensions. Contact is made with perturbative renormalization group calculations for the soft-gap Anderson and Kondo models. A complete description of the quantum critical manyparticle spectra is achieved using suitable marginal operators; technically this can be understood as epsilon-expansion for full many-body spectra.
\end{abstract}

\section{INTRODUCTION}

Zero-temperature phase transitions in quantum impurity models have recently attracted considerable interest (for reviews see Refs. 123 ). These transitions can be observed in systems where a zero-dimensional boundary with internal degrees of freedom (the impurity) interacts with an extended bath of fermions or bosons. Examples of impurity models with non-trivial phase transitions include extensions of the Kondo model where one or two magnetic impurities couple to fermionic baths $\frac{1}{}$, the spinboson model describing a two-level system coupling to a dissipative environment ${ }^{4.5}$, as well as so-called BoseFermi Kondo models for localized spins interacting with both fermionic and bosonic baths. Impurity phase transitions are of relevance for impurities in correlated bulk systems (e.g. superconductors ${ }^{6}$ ), for multilevel impurities like Fullerene molecules $\frac{7}{2}$, as well as for nanodevices like coupled quantum $\operatorname{dots}^{8}$ or point contacts under the influence of dissipative noise ${ }^{9.10}$. In addition, impurity phase transitions have been argued to describe aspects of so-called local quantum criticality in correlated lattice systems. Here, the framework of dynamical meanfield theory is employed to map, e.g., the Kondo lattice model onto a single-impurity Bose-Fermi Kondo model supplemented by self-consistency conditions, for details see Ref. 11.

Diverse techniques have been used to investigate impurity phase transitions, ranging from static and dynamic large- $N$ calculations ${ }^{12}$, conformal field theory ${ }^{3}$, perturbative renormalization group $(\mathrm{RG})^{1,6.13}$ and the localmoment approach ${ }^{14.15}$ to various numerical methods. In particular, significant progress has been made using the numerical renormalization group (NRG) technique, origi- nally developed by Wilson for the Kondo problem ${ }^{16}$. The NRG combines numerically exact diagonalization with the idea of the renormalization group, where progressively smaller energy scales are treated in the course of the calculation. NRG calculations are non-perturbative and are able to access arbitrarily small energies and temperatures. Apart from static and dynamic observables, the NRG provides information about the many-body excitation spectrum of the system at every stage of the RG flow. Thus, it allows to identify fixed points through their fingerprints in the level structure. A detailed understanding of the NRG levels is usually possible if the fixed point can be described by non-interacting bosons or fermions - this is the case for most stable fixed points of impurity models, e.g., the strong-coupling (screened) fixed point of a standard Kondo model. Intermediate-coupling fixed points, usually being interacting, have a completely different NRG level structure, i.e., smaller degeneracies and non-equidistant levels. They cannot be cast into a freeparticle description, with the remarkable exception of the two-channel Kondo fixed point which is known to have a representation in terms of free Majorana fermions 17. In general, the NRG fixed-point spectrum at impurity transitions is fully universal, apart from a non-universal overall prefactor and discretization effects.

The purpose of this paper is to demonstrate that a complete understanding of the NRG many-body spectrum of critical fixed points is actually possible, by utilizing renormalized perturbation theory around a noninteracting fixed point. In the soft-gap Anderson model, this approach can be employed near certain values of the bath exponent which can be identified as critical dimensions. Using the knowledge from perturbative RG calculations, which yield the relevant coupling constants 
being parametrically small near the critical dimension, we can construct the entire quantum critical many-body spectrum from a free-fermion model supplemented by a small perturbation. In other words, we shall perform epsilon-expansions to determine a complete many-body spectrum (instead of certain renormalized couplings or observables). Vice versa, our method allows to identify relevant degrees of freedom and their marginal couplings by carefully analyzing the NRG spectra near critical dimensions of impurity quantum phase transitions.

The paper is organized as follows. In Sec. II we give a brief introduction to the physics of the soft-gap Anderson model and its quantum phase transitions. Sec. III summarizes the recent results from perturbative RG for both the soft-gap Anderson and Kondo models. Section IV describes the numerical renormalization group (NRG) approach which is used here to obtain information about the structure of the quantum critical points. The main part of the paper is Sec. $\nabla$ in which we discuss (i) the numerical data for the structure of the quantum critical points and (ii) the analytical description of these interacting fixed points close to the upper (lower) critical dimension $r=0(r=1 / 2)$. The main conclusions of the paper are summarized in Sec. VI where we also mention other problems for which an analysis of the type presented here might be useful.

\section{SOFT-GAP ANDERSON MODEL}

The Hamiltonian of the soft-gap Anderson mode ${ }^{12}$ is given by:

$$
\begin{aligned}
H= & \varepsilon_{f} \sum_{\sigma} f_{\sigma}^{\dagger} f_{\sigma}+U f_{\uparrow}^{\dagger} f_{\uparrow} f_{\downarrow}^{\dagger} f_{\downarrow} \\
& +\sum_{k \sigma} \varepsilon_{k} c_{k \sigma}^{\dagger} c_{k \sigma}+V \sum_{k \sigma}\left(f_{\sigma}^{\dagger} c_{k \sigma}+c_{k \sigma}^{\dagger} f_{\sigma}\right)
\end{aligned}
$$

This model describes the coupling of electronic degrees of freedom at an impurity site (operators $f_{\sigma}^{(\dagger)}$ ) to a fermionic bath (operators $c_{k \sigma}^{(\dagger)}$ ) via a hybridization $V$. The $f$-electrons are subject to a local Coulomb repulsion $U$, while the fermionic bath consists of a noninteracting conduction band with dispersion $\varepsilon_{k}$. The model eq. (11) has the same form as the single-impurity Anderson model ${ }^{18}$ but for the soft-gap model we require that the hybridization function $\widetilde{\Delta}(\omega)=\pi V^{2} \sum_{k} \delta\left(\omega-\varepsilon_{k}\right)$ has a soft-gap at the Fermi level, $\widetilde{\Delta}(\omega)=\Delta|\omega|^{r}$, with an exponent $r>0$. This translates into a local conduction band density of states $\rho(\omega)=\rho_{0}|\omega|^{r}$ at low energies. In the numerical calculations we used a band where this power law extends over the whole bandwidth $D$, i.e., from $\omega=-D / 2$ to $+D / 2$, and we have $\rho_{0}=(2 / D)^{r+1}(r+1) / 2$. However, the universal lowtemperature physics to be discussed in the following does not depend on the details of the density of states at high energies, and consequently we will use the low-energy prefactor of the density of states, $\rho_{0}$, to represent the

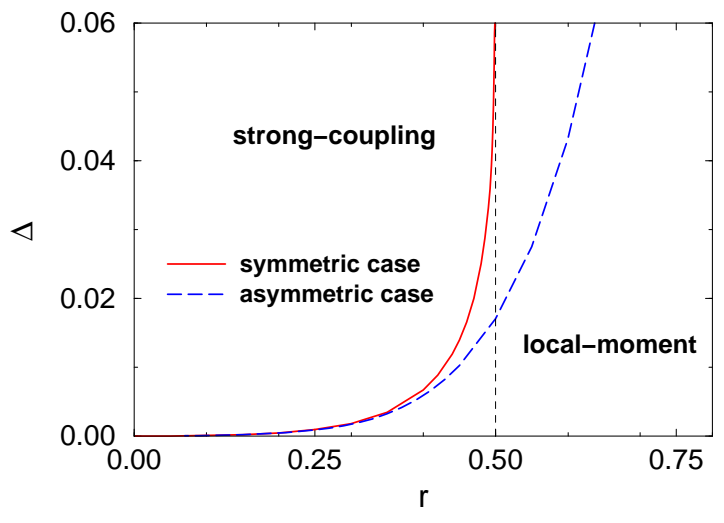

FIG. 1: $T=0$ phase diagram for the soft-gap Anderson model in the p-h symmetric case (solid line, $U=10^{-3}, \varepsilon_{f}=$ $-0.5 \cdot 10^{-3}$, conduction band cutoff at -1 and 1 ) and the p-h asymmetric case (dashed line, $\left.\varepsilon_{f}=-0.4 \cdot 10^{-3}\right) ; \Delta$ measures the hybridization strength, $\widetilde{\Delta}(\omega)=\Delta|\omega|^{r}$.

dimensionful energy scale of the problem. Assuming a particle-hole symmetric band, the model (1) is particlehole symmetric for $\epsilon_{f}=-U / 2$.

The soft-gap Anderson model (1) with $0<r<\infty$ displays a very rich behaviour, in particular a continuous transition between a local-moment (LM) and a strongcoupling (SC) phase. Figure 1 shows a typical phase diagram for the soft-gap Anderson model. In the particlehole (p-h) symmetric case (solid line) the critical coupling $\Delta_{\mathrm{c}}$ diverges at $r=\frac{1}{2}$, and no screening occurs for $r>\frac{1}{2}$ (Refs. 19 20). No divergence occurs for p-h asymmetry (dashed line) 19 .

We now briefly describe the properties of the fixed points in the soft-gap Anderson and Kondo models 19 . Due to the power-law conduction band density of states, already the stable LM and SC fixed points show nontrivial behaviour ${ }^{19.20}$. The LM phase has the properties of a free $\operatorname{spin} \frac{1}{2}$ with residual entropy $S_{\mathrm{imp}}=k_{B} \ln 2$ and low-temperature impurity susceptibility $\chi_{\mathrm{imp}}=$ $1 /\left(4 k_{B} T\right)$, but the leading corrections show $r$-dependent power laws. The p-h symmetric SC fixed point has very unusual properties, namely $S_{\mathrm{imp}}=2 r k_{B} \ln 2, \chi_{\mathrm{imp}}=$ $r /\left(8 k_{B} T\right)$ for $0<r<\frac{1}{2}$. In contrast, the p-h asymmetric SC fixed point simply displays a completely screened moment, $S_{\mathrm{imp}}=T \chi_{\mathrm{imp}}=0$. The impurity spectral function follows a $\omega^{r}$ power law at both the LM and the asymmetric SC fixed point, whereas it diverges as $\omega^{-r}$ at the symmetric SC fixed point - this "peak" can be viewed as a generalization of the Kondo resonance in the standard case $(r=0)$, and scaling of this peak is observed upon approaching the SC-LM phase boundary ${ }^{14,20}$. At the critical point, non-trivial behaviour corresponding to a fractional moment can be observed: $S_{\mathrm{imp}}=k_{B} \mathcal{C}_{S}(r)$, $\chi_{\text {imp }}=\mathcal{C}_{\chi}(r) /\left(k_{B} T\right)$ with $\mathcal{C}_{S}, \mathcal{C}_{\chi}$ being universal functions of $r$ (see Refs. 1921). The spectral functions at the quantum critical points display an $\omega^{-r}$ power law (for $r<1$ ) with a remarkable "pinning" of the critical exponent. 


\section{RESULTS FROM PERTURBATIVE RG}

The Anderson model (11) is equivalent to a Kondo model when charge fluctuations on the impurity site are negligible. The Hamiltonian for the soft-gap Kondo model can be written as

$$
H=J \vec{S} \cdot \overrightarrow{s_{0}}+\sum_{k \sigma} \varepsilon_{k} c_{k \sigma}^{\dagger} c_{k \sigma}
$$

where $\vec{s}(0)=\sum_{k k^{\prime} \sigma \sigma^{\prime}} c_{k \sigma}^{\dagger} \vec{\sigma}_{\sigma \sigma^{\prime}} c_{k^{\prime} \sigma^{\prime}} / 2$ is the conduction electron spin at the impurity site $\mathbf{r}=0$, and the conduction electron density of states follows a power law $\rho(\omega)=\rho_{0}|\omega|^{r}$ as above.

\section{A. RG near $r=0$}

For small values of the density of states exponent $r$, the phase transition in the pseudogap Kondo model can be accessed from the weak-coupling limit, using a generalization of Anderson's poor man's scaling. Power counting about the local moment fixed point (LM) shows that $\operatorname{dim}[J]=-r$, i.e., the Kondo coupling is marginal for $r=0$. We introduce a renormalized dimensionless Kondo coupling $j$ according to

$$
\rho_{0} J=\mu^{-r} j
$$

where $\mu$ plays the role of a UV cutoff. The flow of the renormalized Kondo coupling $j$ is given by the beta function

$$
\beta(j)=r j-j^{2}+\mathcal{O}\left(j^{3}\right)
$$

For $r>0$ there is a stable fixed point at $j^{*}=0$ corresponding to the local-moment phase (LM). An unstable fixed point, controlling the transition to the strongcoupling phase, exists at

$$
j^{*}=r
$$

and the critical properties can be determined in a double expansion in $r$ and $j^{6}$. P-h asymmetry is irrelevant, i.e., a potential scattering term $E$ scales to zero according to $\beta(e)=r e\left(\right.$ where $\rho_{0} E=\mu^{-r} e$ ), thus the above expansion captures the $\mathrm{p}$-h symmetric critical fixed point (SCR). As the dynamical exponent $\nu, 1 / \nu=r+\mathcal{O}\left(r^{2}\right)$, diverges as $r \rightarrow 0^{+}, r=0$ plays the role of a lower-critical dimension of the transition under consideration.

\section{B. RG near $r=1 / 2$}

For $r$ near $1 / 2$ the $\mathrm{p}$-h symmetric critical fixed point moves to strong Kondo coupling, and the language of the $\mathrm{p}$ - $\mathrm{h}$ symmetric Anderson model becomes more appropriate 13 . First, the conduction electrons are integrated out exactly, yielding a self-energy $\Sigma_{f}=V^{2} G_{c 0}$ for the $f$ electrons, where $G_{c 0}$ is the bare conduction electron Green's function at the impurity location. In the low-energy limit the $f$ electron propagator is then given by

$$
G_{f}\left(i \omega_{n}\right)^{-1}=i \omega_{n}-i A_{0} \operatorname{sgn}\left(\omega_{n}\right)\left|\omega_{n}\right|^{r}
$$

where the $\left|\omega_{n}\right|^{r}$ self-energy term dominates for $r<1$, and the prefactor $A_{0}$ is

$$
A_{0}=\frac{\pi \rho_{0} V^{2}}{\cos \frac{\pi r}{2}} .
$$

Equation (6) describes the physics of a non-interacting resonant level model with a soft-gap density of states. Interestingly, the impurity spin is not fully screened for $r>0$, and the residual entropy is $2 r \ln 2$. This precisely corresponds to the symmetric strong-coupling (SC) phase of the soft-gap Anderson and Kondo model ${ }^{19}$.

Dimensional analysis, using $\operatorname{dim}[f]=(1-r) / 2[$ where $f$ represents the dressed fermion according to eq. (6)], now shows that the interaction term $U$ of the Anderson model scales as $\operatorname{dim}[U]=2 r-1$, i.e., it is marginal at $r=1 / 2$. This suggests a perturbative expansion in $U$ around the SC fixed point. We introduce a dimensionless renormalized on-site interaction $u$ via

$$
U=\mu^{2 r-1} A_{0}^{2} u .
$$

The beta funcion receives the lowest non-trivial contribution at two-loop order and reads 13

$$
\beta(u)=(1-2 r) u-\frac{3(\pi-2 \ln 4)}{\pi^{2}} u^{3}+\mathcal{O}\left(u^{5}\right) .
$$

For $r<1 / 2$ a non-interacting stable fixed point is at $u^{*}=$ 0 - this is the symmetric strong-coupling fixed point, it becomes unstable for $r>1 / 2$. Additionally, for $r<1 / 2$ there is a pair of critical fixed points (SCR, SCR') located at $u^{* 2}=\pi^{2}(1-2 r) /[3(\pi-2 \ln 4)]$, i.e.,

$$
u^{*}= \pm 4.22 \sqrt{1 / 2-r} \text {. }
$$

These fixed points describe the transition between an unscreened (spin or charge) moment phase and the symmetric strong-coupling phase 13 .

Summarizing, both (4) and (9) capture the same critical SCR fixed point. This fixed point can be accessed either by an expansion around the weak-coupling LM fixed point, i.e., around the decoupled impurity limit, valid for $r \ll 1$, or by an expansion around the strong-coupling SC fixed point, i.e., around a non-interacting resonant-level (or Anderson) impurity, and this expansion is valid for $1 / 2-r \ll 1$.

\section{NUMERICAL RENORMALIZATION GROUP}

Here we describe the numerical renormalization group method, suitably extended to handle non-constant couplings $\widetilde{\Delta}(\omega)$ (see Refs. 1920,22 ). This method allows a 
non-perturbative calculation of the many-particle spectrum and physical properties in the whole parameter regime of the model eq. (11), in particular in the lowtemperature limit, so that the structure of the quantum critical points is accessible, as discussed in Sec. D

A detailed discussion of how the NRG can be applied to the soft-gap Anderson model can be found in Refs. 19, 20 22. Here we focus on those aspects of the approach necessary to understand how information on the fixed points can be extracted.

The NRG is based on a logarithmic discretization of the energy axis, i.e. one introduces a parameter $\Lambda$ and divides the energy axis into intervals $\left[-\Lambda^{-n},-\Lambda^{-(n+1)}\right]$ and $\left[\Lambda^{-(n+1)}, \Lambda^{-n}\right]$ for $n=0,1, \ldots, \infty$ (see Refs. 16 23). With some further manipulations the original model can be mapped onto a semi-infinite chain with the Hamiltonian

$$
\begin{aligned}
& H=\varepsilon_{f} \sum_{\sigma} f_{\sigma}^{\dagger} f_{\sigma}+U f_{\uparrow}^{\dagger} f_{\uparrow} f_{\downarrow}^{\dagger} f_{\downarrow} \\
& +\sqrt{\frac{\xi_{0}}{\pi}} \sum_{\sigma}\left[f_{\sigma}^{\dagger} c_{0 \sigma}+c_{0 \sigma}^{\dagger} f_{\sigma}\right] \\
& +\sum_{\sigma n=0}^{\infty}\left[\varepsilon_{n} c_{n \sigma}^{\dagger} c_{n \sigma}+t_{n}\left(c_{n \sigma}^{\dagger} c_{n+1 \sigma}+c_{n+1 \sigma}^{\dagger} c_{n \sigma}\right)\right],
\end{aligned}
$$

with

$$
\xi_{0}=\int_{-1}^{1} \mathrm{~d} \omega \widetilde{\Delta}(\omega)
$$

For a p-h symmetric conduction band, all the on-site energies $\varepsilon_{n}$ vanish. If, in addition, the power law in $\widetilde{\Delta}(\omega)$ extends up to a hard cut-off $\omega_{c}$ (we set $\omega_{c}=1$ ), an exact expression for the hopping matrix elements $t_{n}$ can be given $^{20}$ :

$$
\begin{aligned}
t_{n} & =\Lambda^{-n / 2} \frac{r+1}{r+2} \frac{1-\Lambda^{-(r+2)}}{1-\Lambda^{-(r+1)}}\left[1-\Lambda^{-(n+r+1)}\right] \\
& \times\left[1-\Lambda^{-(2 n+r+1)}\right]^{-1 / 2}\left[1-\Lambda^{-(2 n+r+3)}\right]^{-1 / 2}
\end{aligned}
$$

for even $n$ and

$$
\begin{aligned}
t_{n} & =\Lambda^{-(n+r) / 2} \frac{r+1}{r+2} \frac{1-\Lambda^{-(r+2)}}{1-\Lambda^{-(r+1)}}\left[1-\Lambda^{-(n+1)}\right] \\
& \times\left[1-\Lambda^{-(2 n+r+1)}\right]^{-1 / 2}\left[1-\Lambda^{-(2 n+r+3)}\right]^{-1 / 2}
\end{aligned}
$$

for odd $n$. The semi-infinite chain is solved iteratively by starting from the impurity and successively adding chain sites. As the coupling $t_{n}$ between two adjacent sites $n$ and $n+1$ decreases as $\Lambda^{-n / 2}$ for large $n$, the low-energy states of the chain with $n+1$ sites are generally determined by a comparatively small number $N_{\mathrm{S}}$ of states close to the ground state of the $n$-site system. In practice one retains only these $N_{\mathrm{s}}$ states from the $n$-site chain to set

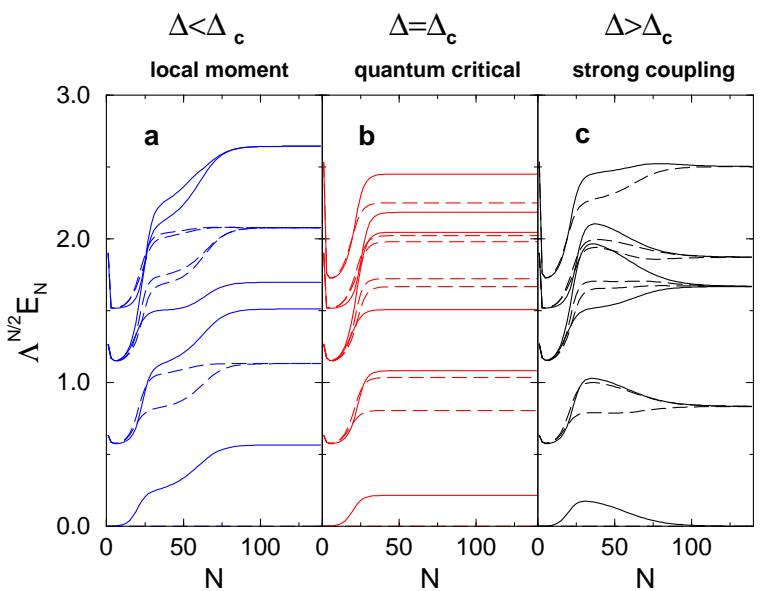

FIG. 2: Flow diagrams for the low-energy many-body excitations obtained from the numerical renormalization group for the three different fixed points of the p-h symmetric softgap Anderson model (exponent $r=0.4$ ). $N$ is the number of iterations of the NRG procedure, $\Lambda$ the NRG discretization parameter. Solid lines: $(Q, S)=(1,0)$, dashed lines: $(Q, S)=(0,1 / 2)$.

up the Hilbert space for the $n+1$ site chain, thus preventing the usual exponential growth of the Hilbert space as $n$ increases. Eventually, after $n_{\mathrm{NRG}}$ sites have been included in the calculation, addition of another site will not change significantly the spectrum of many-particle excitations; the spectrum is very close to that of the fixed point, and the calculation may be terminated.

In this way, the NRG iteration gives the many-particle energies $E_{N}$ for a sequence of Hamiltonians $H_{N}$ which correspond to the Hamiltonian eq. (11) by the replacement

$$
\sum_{\sigma n=0}^{\infty} \longrightarrow \sum_{\sigma n=0}^{N-1}
$$

An example for the dependence of the lowest lying energy levels on the chain length (the flow diagram) is given in Fig. 2r for the soft-gap Anderson model with $r=0.4, D=2, U / D=10^{-3}$ and $\Delta=0.0075$; the parameters used for the NRG calculations are $\Lambda=2$ and $N_{\mathrm{s}}=300$. The states are labelled by the quantum numbers $Q$ (which characterizes the number of particles measured relative to half-filling), and the total spin, $S$ [solid lines in Fig. 2 are for $(Q, S)=(1,0)$, dashed lines for $(Q, S)=(0,1 / 2)]$. As mentioned above, the energy scale is reduced in each step by a factor $\Lambda^{1 / 2}$. To allow for a direct comparison of the energies for different chain lengths, it is thus convenient to plot $\Lambda^{N / 2} E_{N}$ instead of the eigenvalues $E_{N}$ of the $N$-site chain directly. Note that here and in the following we use the convention that the energies shown in the flow diagrams are proportional to the bandwidth $D$.

As is apparent from Fig. 2r, the properties of the system in this case do not change further for chain lengths $n_{\mathrm{NRG}}>120$. Without going into details now, one can 


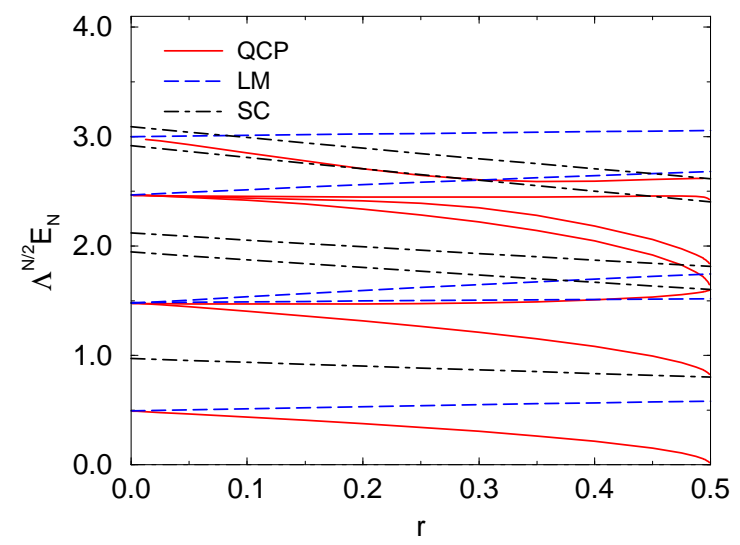

FIG. 3: Dependence of the many-particle spectra for the three fixed points of the p-h symmetric soft-gap Anderson model on the exponent $r$ : SC (black dot-dashed lines), LM (blue dashed lines), and the (symmetric) quantum critical point (red solid lines). The data are shown for the subspace $Q=1$ and $S=0$ only.

show that the distribution of energy levels for $N>120$ in Fig. 2. is characteristic of the SC phase of the model (see Sec. V].

If by contrast we choose instead a value of $\Delta=0.006$, we obtain the flow diagram shown in Fig. 22 . Here it is evident that the fixed point level structure is entirely different from the SC solution, and indeed this particular pattern is now characteristic of the LM phase of the model. We can thus conclude, simply from inspection of the two flow diagrams, that the critical $\Delta_{\mathrm{c}}$ separating the SC and LM phases of the soft-gap Anderson model for the model parameters specified, lies in the interval $[0.006,0.0075]$.

Tuning the value of $\Delta$ to the critical value $\Delta_{c}$, results in the flow diagram of Fig. 20. Apparently, the structure of the fixed point at $\Delta_{c}$ neither coincides with the SC nor with the LM fixed point. It is clear that it cannot be simply constructed from single-particle states as for the $\mathrm{SC}$ and LM fixed points. An important observation is that certain degeneracies present in the SC or LM fixed points are lifted at the QCP. As shown in the following section, a further hint on the structure of the QCPs is given by the dependence of their many-particle spectra on the bath exponent $r$.

\section{STRUCTURE OF THE QUANTUM CRITICAL POINTS}

In Fig. 3 the many-particle spectra of the three fixed points (SC: dot-dashed lines, LM: dashed lines, and QCP: solid lines) of the symmetric soft-gap model are plotted as functions of the exponent $r$ (for a similar figure, see Fig. 13 in Ref. 19). The data are shown for an odd number of sites only and we select the lowest lying energy levels for the subspace $Q=1$ and $S=0$.

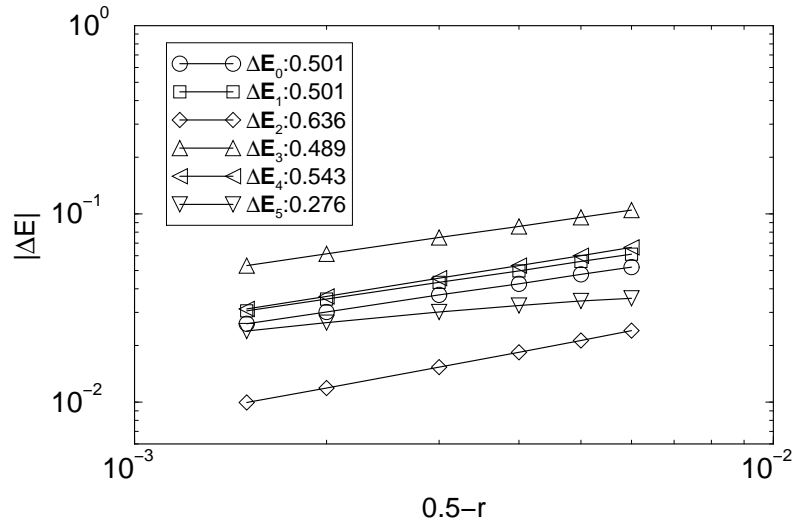

FIG. 4: Difference $\Delta E$ between the energy levels of QCP and SC fixed points close to $r=1 / 2$ in a double-logarithmic plot. The inset shows the values of the exponents obtained from a fit to the data points.

As usual, the fixed point structure of the strong coupling and local moment phases can be easily constructed from the single-particle states of a free conduction electron chain. This is discussed in more detail later. Let us now turn to the line of quantum critical points. What information can be extracted from Fig. 3 to understand the structure of these fixed points?

First we observe that the levels of the quantum critical points, $E_{N, \mathrm{QCP}}(r)$, approach the levels of the LM (SC) fixed points in the limit $r \rightarrow 0(r \rightarrow 1 / 2)$ :

$$
\begin{aligned}
\lim _{r \rightarrow 0}\left\{E_{N, \mathrm{QCP}}(r)\right\} & =\left\{E_{N, \mathrm{LM}}(r=0)\right\}, \\
\lim _{r \rightarrow 1 / 2}\left\{E_{N, \mathrm{QCP}}(r)\right\} & =\left\{E_{N, \mathrm{SC}}(r=1 / 2)\right\},
\end{aligned}
$$

where $\{\ldots\}$ denotes the whole set of many-particle states.

For $r \rightarrow 0$, each individual many-particle level $E_{N, \mathrm{QCP}}(r)$ deviates linearly from the levels of the LM fixed point, while the deviation from the SC levels is proportional to $\sqrt{1 / 2-r}$ for $r \rightarrow 1 / 2$. This is illustrated in Fig. 4 where we plot a selection of energy differences $\Delta E$ between levels of QCP and SC fixed points close to $r=1 / 2$. The inset shows the values of the exponents obtained from a fit to the data points. For some levels, there are significant deviations from the exponent $1 / 2$. This is because the correct exponent is only obtained in the limit $r \rightarrow 1 / 2$ (the QCP levels have been obtained only up to $r=0.4985$ ).

Note that the behaviour of the many-particle levels close to $r=1 / 2$ has direct consequences for physical properties at the QCP; the critical exponent of the local susceptibility at the QCP, for example, shows a squareroot dependence on $(1 / 2-r)$ close to $r=1 / 2$, see Ref. 19 .

In both limits, $r \rightarrow 0$ and $r \rightarrow 1 / 2$, we observe that degeneracies due to the combination of single-particle levels, present at the LM and SC fixed points, are lifted at the quantum critical fixed points as soon as one is moving away from $r=0$ and $r=1 / 2$, respectively. This already suggests that the quantum critical point is inter- 
acting and cannot be constructed from non-interacting single-particle states.

In the remainder of the paper we want to show how to connect this information from NRG to the perturbative RG of Sec. [III We know that the critical fixed point can be accessed via two different epsilon-expansions.6.13 near the two critical dimensions, and, combined with renormalized perturbation theory, these expansions can be used to evaluate various observables near criticality. Here, we will employ this concept to perform renormalized perturbation theory for the entire many-body spectrum at the critical fixed point. To do so, we will start from the many-body spectrum of one of the trivial fixed points, i.e., LM near $r=0$ and SC near $r=1 / 2$, and evaluate corrections to it in lowest-order perturbation theory. This will be done within the NRG concept working directly with the discrete many-body spectra corresponding to a finite NRG chain (which is diagonalized numerically). As the relevant energy scale of the spectra decreases as $\Lambda^{-n / 2}$ along the NRG iteration, the strength of the perturbation has to be scaled as well, as the goal is to capture a fixed point of the NRG method. This scaling of the perturbation follows precisely from its scaling dimension - the perturbation marginal at the value of $r$ corresponding to the critical dimension. With the proper scaling, the operator which we use to capture the difference between the free-fermion and critical fixed points becomes exactly marginal [see eqs. (21) and (37) below].

\section{A. Perturbation theory close to $r=0$}

Let us now describe in detail the analysis of the deviation of the QCP levels from the LM levels close to $r=0$ (the case $r=1 / 2$ is discussed in Sec. $\mathrm{\nabla B}$ ). An effective description of the LM fixed point is given by a finite chain with the impurity decoupled from the conduction electron part, see Fig. [5

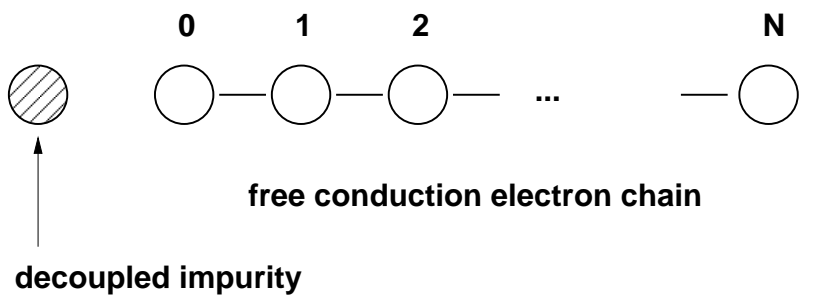

FIG. 5: The spectrum of the LM fixed point is described by the impurity decoupled from the free conduction electron chain.

The conduction electron part of the effective Hamiltonian is given by

$$
H_{\mathrm{c}, N}=\sum_{\sigma n=0}^{N-1} t_{n}\left(c_{n \sigma}^{\dagger} c_{n+1 \sigma}+c_{n+1 \sigma}^{\dagger} c_{n \sigma}\right) .
$$

As usual, the structure of the fixed point spectra depends on whether the total number of sites is even or odd. To simplify the discussion in the following, we only consider a total odd number of sites (the flow diagrams of Fig. 2 are all calculated for this case). For the LM fixed point, this means that the number of sites, $N+1$, of the free conduction electron chain is even, so $N$ in eq. (17) is odd.

The single-particle spectrum of the free chain with an even number of sites, corresponding to the diagonalized Hamiltonian

$$
\bar{H}_{\mathrm{c}, N}=\sum_{\sigma p} \epsilon_{p} \xi_{p \sigma}^{\dagger} \xi_{p \sigma}
$$

is sketched in Fig. [6 (Note that the $\epsilon_{p}$ have to be evaluated numerically.)

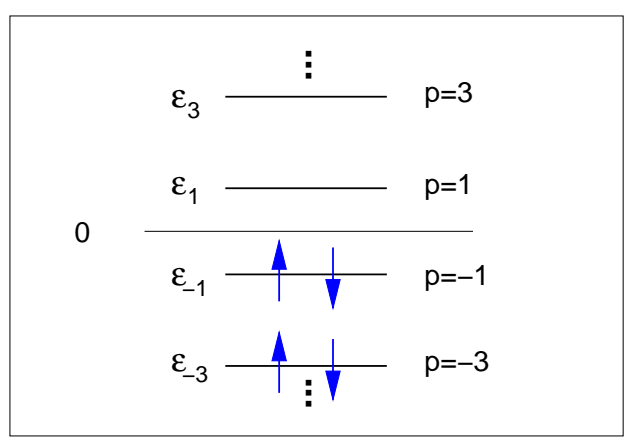

FIG. 6: Single-particle spectrum of the free conduction electron chain eq. (18). The ground state is given by all the levels with $p<0$ filled.

As we assume p-h symmetry, the positions of the single-particle levels are symmetric with respect to 0 with

$$
\epsilon_{p}=-\epsilon_{-p} \quad, \quad p=1,3, \ldots, N,
$$

and

$$
\sum_{p} \equiv \sum_{p=-N, p \text { odd }}^{p=N}
$$

Note that an equally spaced spectrum of single-particle levels is only recovered in the limit $\Lambda \rightarrow 1$ (see Fig. 6 in Ref. 17) for the case $r=0$.

The RG analysis of Sec. III tells us that the critical fixed point is perturbative accessible from the LM one using a Kondo-type coupling as perturbation. We thus focus on the operator

$$
H_{N}^{\prime}=\alpha(r) f(N) \vec{S}_{\mathrm{imp}} \cdot \vec{s}_{0},
$$

with the goal to calculate the many-body spectrum of the critical fixed point via perturbation theory in $H_{N}^{\prime}$ for small $r$. The function $\alpha(r)$ contains the fixed-point value of the Kondo-type coupling, and $f(N)$ will be chosen such that $H_{N}^{\prime}$ is exactly marginal, i.e., the effect of $H_{N}^{\prime}$ 
on the many-particle energies decreases as $\Lambda^{-N / 2}$ which is the same $N$ dependence which governs the scaling of the many-particle spectrum itself. The scaling analysis of Sec. III] eqs. (3) and (5), suggests a parametrization of the coupling as

$$
\alpha(r)=\frac{\mu^{-r}}{\rho_{0}} \alpha r
$$

where $\rho_{0}$ is the prefactor in the density of states, and $\mu$ is a scale of order of the bandwidth - such a factor is required here to make $\alpha$ a dimensionless parameter. Thus, the strength of the perturbation increases linearly with $r$ at small $r$ (where $\mu^{-r} / \rho_{0}=D+\mathcal{O}(r)$ for a featureless $|\omega|^{r}$ density of states).

The qualitative influence of the operator $\vec{S}_{\mathrm{imp}} \cdot \vec{s}_{0}$ on the many-particle states has been discussed in general in Ref. 19 for finite $r$ and in Refs. 16.23 for $r=0$. Whereas an antiferromagnetic exchange coupling is marginally relevant in the gapless case $(r=0)$, it turns out to be irrelevant for finite $r$, see Ref. 19. This is of course consistent with the scaling analysis of Sec. IIII the operator (21) simply represents a Kondo coupling, with a tree-level scaling dimension of $\operatorname{dim}[J]=-r$. A detailed analysis of the $N$-dependence of the operator $\vec{S}_{\mathrm{imp}} \cdot \vec{s}_{0}$ shows that it decreases as $\Lambda^{-N r / 2} \Lambda^{-N / 2}=\Lambda^{-N(r+1) / 2}$ with increasing $N$. Consequently, we have to choose

$$
f(N)=\Lambda^{N r / 2} \text {. }
$$

This result also directly follows from $\operatorname{dim}[J]=-r$ : As the NRG discretization yields a decrease of the running energy scale of $\Lambda^{-N / 2}$, the $\vec{S}_{\text {imp }} \cdot \vec{s}_{0}$ term in $H_{N}^{\prime}$ (21) scales as $\Lambda^{-N r / 2}$. The function $f(N)$ is now simply chosen to compensate this effect; using eq. (23) the operator $H_{N}^{\prime}$ becomes exactly marginal.

Now we turn to a discussion of the many-body spectrum. The relevant ground state of the effective model for the LM fixed point consists of the filled impurity level (with one electron with either spin $\uparrow$ or $\downarrow$ ) and all the conduction electron states with $p<0$ filled with both $\uparrow$ and $\downarrow$, as shown in Fig. [6] Let us now focus on excitations with energy $\epsilon_{1}+\epsilon_{3}$ measured with respect to the ground state. Figure 7 shows one such excitation; in this case, one electron with spin $\downarrow$ is removed from the $p=-3$ level and one electron with spin $\downarrow$ is added to the $p=1$ level. The impurity level is assumed to be filled with an electron with spin $\uparrow$, so the resulting state has $Q=0$ and $S_{z}=+1 / 2$. In total, there are 32 states with excitation energy $\epsilon_{1}+\epsilon_{3}$. These states can be classified using the quantum numbers $Q, S$, and $S_{z}$.

Here we consider only the states with quantum numbers $Q=0, S=1 / 2$, and $S_{z}=1 / 2$ (with excitation energy $\epsilon_{1}+\epsilon_{3}$ ) which form a four-dimensional subspace. As the state shown in Fig. 7 is not an eigenstate of the total spin $S$, we have to form proper linear combinations to obtain a basis for this subspace; this basis can be written in the form:

$\left|\psi_{1}\right\rangle=\frac{1}{\sqrt{2}} f_{\uparrow}^{\dagger}\left(\xi_{1 \uparrow}^{\dagger} \xi_{-3 \uparrow}+\xi_{1 \downarrow}^{\dagger} \xi_{-3 \downarrow}\right)\left|\psi_{0}\right\rangle$

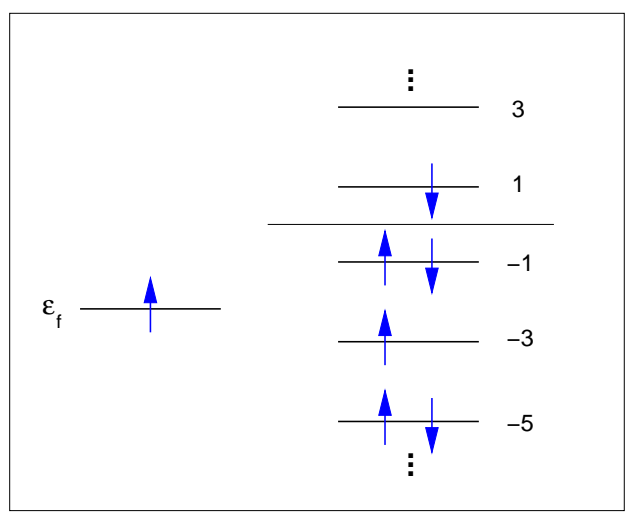

FIG. 7: One possible excitation with energy $\epsilon_{1}+\epsilon_{3}$ and quantum numbers $Q=0$ and $S_{z}=+1 / 2$.

$$
\begin{aligned}
\left|\psi_{2}\right\rangle & =\left[\frac{1}{\sqrt{6}} f_{\uparrow}^{\dagger}\left(\xi_{1 \uparrow}^{\dagger} \xi_{-3 \uparrow}-\xi_{1 \downarrow}^{\dagger} \xi_{-3 \downarrow}\right)+\frac{2}{\sqrt{6}} f_{\downarrow}^{\dagger} \xi_{1 \uparrow}^{\dagger} \xi_{-3 \downarrow}\right]\left|\psi_{0}\right\rangle \\
\left|\psi_{3}\right\rangle & =\frac{1}{\sqrt{2}} f_{\uparrow}^{\dagger}\left(\xi_{3 \uparrow}^{\dagger} \xi_{-1 \uparrow}+\xi_{3 \downarrow}^{\dagger} \xi_{-1 \downarrow}\right)\left|\psi_{0}\right\rangle \\
\left|\psi_{4}\right\rangle & =\left[\frac{1}{\sqrt{6}} f_{\uparrow}^{\dagger}\left(\xi_{3 \uparrow}^{\dagger} \xi_{-1 \uparrow}-\xi_{3 \downarrow}^{\dagger} \xi_{-1 \downarrow}\right)+\frac{2}{\sqrt{6}} f_{\downarrow}^{\dagger} \xi_{3 \uparrow}^{\dagger} \xi_{-1 \downarrow}\right]\left|\psi_{0}\right\rangle
\end{aligned}
$$

where the state $\left|\psi_{0}\right\rangle$ is given by the product of the ground state of the conduction electron chain and the empty impurity level:

$$
\left|\psi_{0}\right\rangle=\left[\prod_{p<0} \xi_{p \uparrow}^{\dagger} \xi_{p \downarrow}^{\dagger}|0\rangle_{\mathrm{cond}}\right] \otimes|0\rangle_{\mathrm{imp}} .
$$

The fourfold degeneracy of the subspace $(Q=0$, $\left.S=1 / 2, S_{z}=1 / 2\right)$ of the LM fixed point at energy $\epsilon_{1}+\epsilon_{3}$ is partially split for finite $r$ in the spectrum of the quantum critical fixed point. Let us now calculate the influence of the perturbation $H_{N}^{\prime}$ on the states $\left|\psi_{1}\right\rangle, \ldots\left|\psi_{4}\right\rangle$, concentrating on the splitting of the energy levels up to first order. Degenerate perturbation theory requires the calculation of the matrix

$$
W_{i j}=\left\langle\psi_{i}\left|H_{N}^{\prime}\right| \psi_{j}\right\rangle, \quad i, j=1, \ldots 4,
$$

and a subsequent calculation of the eigenvalues of $\left\{W_{i j}\right\}$ gives the level splitting.

Details of the calculation of the matrix elements $W_{i j}$ are given in Appendix $\mathrm{A}$ The result is

$$
\left\{W_{i j}\right\}=\alpha(r) f(N)\left[\begin{array}{cccc}
0 & \frac{\sqrt{3}}{4} \gamma & 0 & 0 \\
\frac{\sqrt{3}}{4} \gamma & -\frac{1}{2} \beta & 0 & 0 \\
0 & 0 & 0 & \frac{\sqrt{3}}{4} \gamma \\
0 & 0 & \frac{\sqrt{3}}{4} \gamma & -\frac{1}{2} \beta
\end{array}\right],
$$

with $\gamma=\left[\left|\alpha_{01}\right|^{2}-\left|\alpha_{0-3}\right|^{2}\right]$ and $\beta=\left[\left|\alpha_{01}\right|^{2}+\left|\alpha_{0-3}\right|^{2}\right]$. The $N$-dependence of the coefficients $\alpha_{0 p}$ (which relate 
the operators $c_{0 \sigma}$ and $\xi_{p \sigma}$, see eq. (A8)) is given by

$$
\left|\alpha_{0 p}\right|^{2} \propto \Lambda^{-N r / 2} \Lambda^{-N / 2}
$$

(see also Sec. III A in Ref. 19). Numerically we find that

$$
\begin{aligned}
& \gamma=-0.1478 \cdot \Lambda^{-N r / 2} \Lambda^{-N / 2} \\
& \beta=2.0249 \cdot \Lambda^{-N r / 2} \Lambda^{-N / 2}
\end{aligned}
$$

where the prefactors depend on the exponent $r$ and the discretization parameter $\Lambda$ (the quoted values are for $r=$ 0.01 and $\Lambda=2.0)$. The matrix $\left\{W_{i j}\right\}_{r=0.01}$ then takes the form

$$
\begin{aligned}
& \left\{W_{i j}\right\}_{r=0.01}=\alpha(r=0.01) \Lambda^{-N / 2} \\
& \quad \times\left[\begin{array}{cccc}
0 & -0.064 & 0 & 0 \\
-0.064 & -1.013 & 0 & 0 \\
0 & 0 & 0 & -0.064 \\
0 & 0 & -0.064 & -1.013
\end{array}\right] .
\end{aligned}
$$

Diagonalization of this matrix gives the first-order corrections to the energy levels

$$
\begin{aligned}
\Delta E_{1}(r=0.01) & =\Delta E_{3}(r=0.01) \\
& =\alpha(r=0.01) \Lambda^{-N / 2} \cdot(-1.0615) \\
\Delta E_{2}(r=0.01) & =\Delta E_{4}(r=0.01) \\
& =\alpha(r=0.01) \Lambda^{-N / 2} \cdot 0.0004
\end{aligned}
$$

with

$$
\begin{aligned}
E_{N, \mathrm{QCP}}(r=0.01, i) & =E_{N, \mathrm{LM}}(r=0.01, i) \\
& +\Delta E_{i}(r=0.01),
\end{aligned}
$$

$(i=1, \ldots 4)$. Apparently, the fourfold degeneracy of the subspace $\left(Q=0, S=1 / 2, S_{z}=1 / 2\right)$ with energy $\epsilon_{1}+\epsilon_{3}$ is split in two levels which are both twofold degenerate.

We repeated this analysis for a couple of other subspaces and a list of the resulting matrices $\left\{W_{i j}\right\}$ and the energy shifts $\Delta E$ is given in Appendix A

Let us now proceed with the comparison of the perturbative results with the structure of the quantum critical fixed point calculated from the NRG. For our specific choice of the conduction band density of states, the relation (22) yields $\alpha(r)=\alpha r D$ for small $r$ (where $\mu^{r} \approx 1$ ). Using the corresponding equations for the energy shifts in Appendix A we observe that a single parameter $\alpha$ must be sufficient to describe the level shifts in all subspaces, provided that the exponent $r$ is small enough so that the perturbative calculations are still valid. A numerical fit gives $\alpha \approx 1.03$ for $\Lambda=2.0$, (the $\Lambda$-dependence of $\alpha$ is discussed later, see Fig. 9).

Figure 8 summarizes the NRG results together with the perturbative analysis for exponents $r$ close to 0 . A flow diagram of the lowest lying energy levels is shown in Fig. 87 for a small value of the exponent, $r=0.03$, so that the levels of the QCP only slightly deviate from
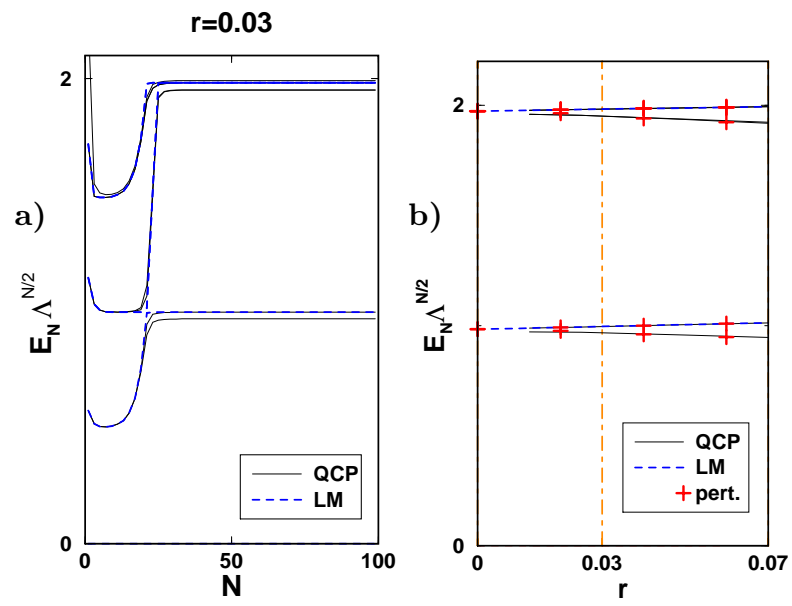

FIG. 8: a) Flow diagram of the lowest lying energy levels for $r=0.03$; dashed lines: flow to the LM fixed point; solid lines: flow to the quantum critical fixed point. b) The deviation of the QCP levels from the LM levels increases linearly with $r$. This deviation together with the splitting of the energy levels can be explained by the perturbative calculation (crosses) as described in the text.

those of the LM fixed point. As discussed above, the deviation of the QCP levels from the LM levels increases linearly with $r$, see Fig. 8 $\mathrm{b}$. We indeed get a very good agreement between the perturbative result (crosses) and the NRG-data (lines) for exponents up to $r \approx 0.07$. The data shown here are for the subspaces $(Q=0, S=1 / 2$, $S_{z}=1 / 2$ ) and energy $2 \epsilon_{1}$ (the levels at $E_{N} \Lambda^{N / 2} \approx 1$, see Appendix A1) and $\left(Q=0, S=1 / 2, S_{z}=1 / 2\right)$ and energy $\epsilon_{1}+\epsilon_{3}$ (the levels at $E_{N} \Lambda^{N / 2} \approx 2$, see the example discussed in this section).

In the NRG, the continuum limit corresponds to the limit $\Lambda \rightarrow 1$, but due to the drastically increasing numerical effort upon reducing $\Lambda$, results for the continuum limit have to be obtained via extrapolation of NRG data for $\Lambda$ in, for example, the range $1.5<\Lambda<3.0$. Figure 9 shows the numerical results from the NRG calculation together with a linear fit to the data: $\alpha(\Lambda)=$ $0.985+0.045(\Lambda-1.0)$. Taking into account the increasing error bars for smaller values of $\Lambda$, the extrapolated value $\alpha(\Lambda \rightarrow 1) \approx 0.985$ is in excellent agreement with the result from the perturbative RG calculation, which is directly for the continuum limit and gives $\alpha=1.0$.

\section{B. Perturbation theory close to $r=1 / 2$}

To describe the deviation of the QCP levels from the $\mathrm{SC}$ levels close to $r=1 / 2$, we have to start from an effective description of the SC fixed point. This is given by a finite chain including the impurity site with the Coulomb repulsion $U=0$ at the impurity site and a hybridization $\bar{V}$ between impurity and the first conduction electron site, see Fig. 10]

Note that the SC fixed point can also be described by 


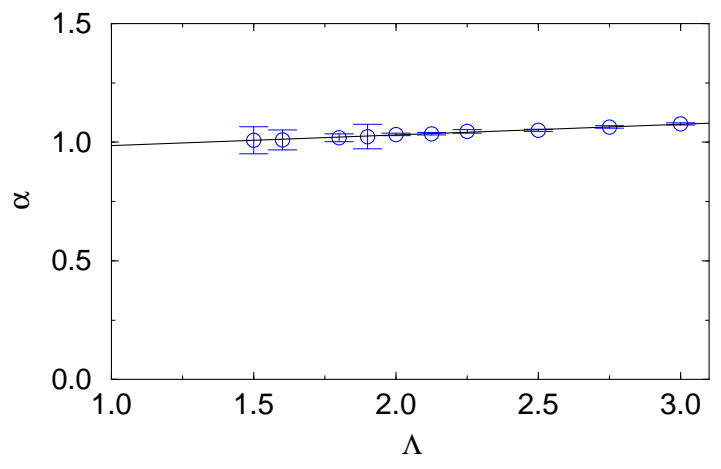

FIG. 9: Dependence of the coupling parameter $\alpha$ on the NRGdiscretization parameter $\Lambda$. The circles correspond to the NRG data and the solid line is a linear fit to the data: $\alpha(\Lambda)=$ $0.985+0.045(\Lambda-1.0)$.

the limit $\bar{V} \rightarrow \infty$ and finite $U$ which means that impurity and first conduction electron site are effectively removed from the chain. This reduces the number of sites of the chain by two and leads to exactly the same level structure as including the impurity with $U=0$. However, the description with the impurity included (and $U=0$ ) is more suitable for the following analysis.

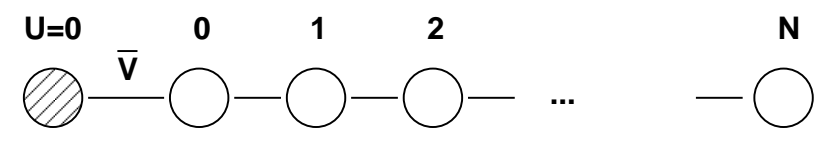

FIG. 10: The spectrum of the SC fixed point is described by the non-interacting impurity coupled to the free conduction electron chain.

The corresponding effective Hamiltonian is that of a soft-gap Anderson model on a finite chain with $N+2$ sites and $\varepsilon_{f}=U=0$ (i.e., a p-h symmetric resonant level model).

$$
H_{\mathrm{sc}, N}=\bar{V} \sum_{\sigma}\left[f_{\sigma}^{\dagger} c_{0 \sigma}+c_{0 \sigma}^{\dagger} f_{\sigma}\right]+H_{\mathrm{c}, N}
$$

with $H_{\mathrm{c}, N}$ as in eq. (17).

As for the effective description of the LM fixed point, the effective Hamiltonian is that of a free chain. Focussing, as above, on odd values of $N$, the total number of sites of this chain, $N+2$, is odd. The single-particle spectrum of the free chain with an odd number of sites, corresponding to the diagonalized Hamiltonian

$$
\bar{H}_{\mathrm{sc}, N}=\sum_{\sigma l} \epsilon_{l} \xi_{l \sigma}^{\dagger} \xi_{l \sigma}
$$

is sketched in Fig. 11] As we assume p-h symmetry, the positions of the single-particle levels are symmetric with respect to 0 with

$$
\epsilon_{0}=0, \quad \epsilon_{l}=-\epsilon_{-l}, \quad l=2,4, \ldots,(N+1),
$$

and

$$
\sum_{l} \equiv \sum_{l=-(N+1), l \text { even }}^{l=N+1} .
$$

The ground state of the effective model for the SC fixed point is fourfold degenerate, with all levels with $l<0$ filled and the level $l=0$ either empty, singly ( $\uparrow$ or $\downarrow$ ) or doubly occupied.

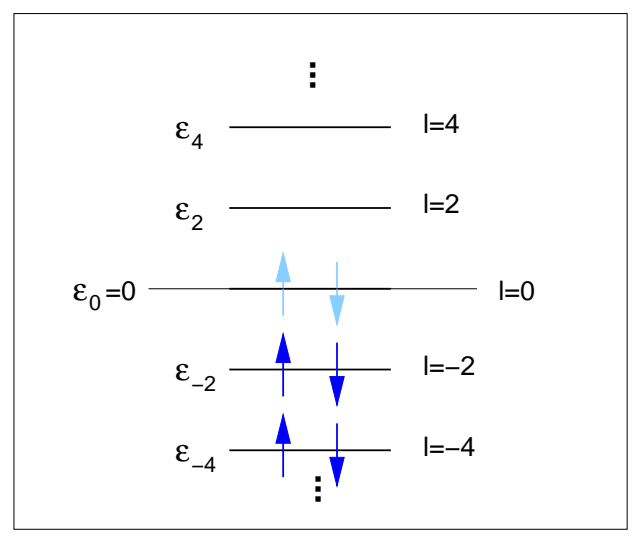

FIG. 11: Single-particle spectrum of the free conduction electron chain eq. (34). The ground state is fourfold degenerate with all the levels with $l<0$ filled and the level $l=0$ either empty, singly ( $\uparrow$ or $\downarrow$ ) or doubly occupied.

According to Sec. III the proper perturbation to access the critical fixed point from the SC one is an on-site repulsion, thus we choose

$$
H_{N}^{\prime}=\beta(r) \bar{f}(N)\left(n_{f \uparrow}-\frac{1}{2}\right)\left(n_{f \downarrow}-\frac{1}{2}\right),
$$

$\left(n_{f \sigma}=f_{\sigma}^{\dagger} f_{\sigma}\right)$ with the strength of the perturbation parametrized as

$$
\beta(r)=\mu^{2 r-1} \rho_{0}^{2} \bar{V}^{4} \beta \sqrt{1 / 2-r} .
$$

see Sec. III Note that $\rho_{0}^{2}(r=1 / 2)=9 /\left(2 D^{3}\right)$ for a featureless power-law density of states with bandwidth $D$. The $N$ dependence of the operator $\left(n_{f \uparrow}-\frac{1}{2}\right)\left(n_{f \downarrow}-\frac{1}{2}\right)$ is given by $\Lambda^{(r-1 / 2) N} \Lambda^{-N / 2}=\Lambda^{(r-1) N}$, so we have to choose

$$
\bar{f}(N)=\Lambda^{(1 / 2-r) N} .
$$

This again follows from the scaling analysis of Sec. III] the on-site repulsion has scaling dimension $\operatorname{dim}[U]=2 r-$ 1. Thus the $\left(n_{f \uparrow}-\frac{1}{2}\right)\left(n_{f \downarrow}-\frac{1}{2}\right)$ term in $H_{N}^{\prime}$ (37) scales as $\Lambda^{N(r-1 / 2)}$, and $\bar{f}(N)$ (39) compensates this behavior to make $H_{N}^{\prime}$ exactly marginal. 
We continue with analyzing the low-lying many-body levels. Similar as above, we focus on one specific example, these are excitations with energy $2 \varepsilon_{2}$ measured with respect to the ground state and quantum numbers $Q=-1$, $S=0$, and $S_{z}=0$. This subspace is two-dimensional and the basis is given by

$$
\begin{aligned}
& \left|\psi_{1}\right\rangle=-\xi_{0 \uparrow}^{\dagger} \xi_{0 \downarrow}^{\dagger} \xi_{-2 \uparrow} \xi_{-2 \downarrow}\left|\psi_{0}\right\rangle, \\
& \left|\psi_{2}\right\rangle=\frac{1}{\sqrt{2}}\left(\xi_{2 \uparrow}^{\dagger} \xi_{-2 \uparrow}+\xi_{2 \downarrow}^{\dagger} \xi_{-2 \downarrow}\right)\left|\psi_{0}\right\rangle
\end{aligned}
$$

with

$$
\left|\psi_{0}\right\rangle=\prod_{l<0} \xi_{l \uparrow}^{\dagger} \xi_{l \downarrow}^{\dagger}|0\rangle
$$

(Note that in this definition of $\left|\psi_{0}\right\rangle$, the $l=0$-level is empty.)

The two-fold degeneracy of this subspace is lifted for $r<1 / 2$ in the spectrum of the quantum critical points. The matrix $W_{i j}=\left\langle\psi_{i}\left|H_{N}^{\prime}\right| \psi_{j}\right\rangle(i, j=1,2)$ is given by

$$
\left\{W_{i j}\right\}=\beta(r) \bar{f}(N)\left|\alpha_{f 2}\right|^{4}\left[\begin{array}{cc}
2-2 \kappa+\kappa^{2} & 2 \sqrt{2} \kappa \\
2 \sqrt{2} \kappa & 2+\kappa^{2}
\end{array}\right],
$$

with $\kappa=\left|\alpha_{f 0}\right|^{2} /\left|\alpha_{f 2}\right|^{2}$. The $N$-dependence of the coefficients $\left|\alpha_{f l}\right|$ (which relate the operators $f_{\mu}$ and $\xi_{l \mu}$, see eq. (B4) is given by

$$
\left|\alpha_{f l}\right|^{2} \propto \Lambda^{(r-1) N / 2},
$$

Numerically we find that

$$
\begin{aligned}
& \left|\alpha_{f 2}\right|^{2}=0.0366 \cdot(D / \bar{V})^{2} \Lambda^{(r-1) N / 2} \\
& \left|\alpha_{f 0}\right|^{2}=0.0930 \cdot(D / \bar{V})^{2} \Lambda^{(r-1) N / 2},
\end{aligned}
$$

where the prefactors depend on the exponent $r$ and the quoted value is for $r=0.499$. The matrix $\left\{W_{i j}\right\}_{r=0.499}$ then takes the form

$$
\begin{aligned}
\left\{W_{i j}\right\}_{r=0.499} & =\beta(r=0.499)(D / \bar{V})^{4} \Lambda^{-N / 2} \\
& \times\left[\begin{array}{cc}
0.0044 & 0.0094 \\
0.0094 & 0.011
\end{array}\right]
\end{aligned}
$$

Diagonalization of this matrix gives the first-order corrections to the energy levels

$$
\begin{aligned}
& \Delta E_{1}(r=0.499)=\beta(r=0.499)(D / \bar{V})^{4} \Lambda^{-N / 2} \cdot(-0.0023) \\
& \Delta E_{2}(r=0.499)=\beta(r=0.499)(D / \bar{V})^{4} \Lambda^{-N / 2} \cdot(0.018)
\end{aligned}
$$

with

$$
\begin{aligned}
E_{N, \mathrm{QCP}}(r & =0.499, i)= \\
E_{N, \mathrm{SC}}(r & =0.499, i)+\Delta E_{i}(r=0.499),
\end{aligned}
$$

$(i=1,2)$. We repeated this analysis for a couple of other subspaces and a list of the resulting matrices $\left\{W_{i j}\right\}$ and the energy shifts $\Delta E$ is given in Appendix B

The comparison of the perturbative results with the numerical results from the NRG calculation is shown in Fig. [12]. As for the case $r \approx 0$ we observe that a single parameter $\beta$ is sufficient to describe the level shifts in all subspaces, provided the exponent $r$ is close enough to $r=1 / 2$ so that the perturbative calculations are valid. For $\Lambda=2.0$ we find $\beta \approx 70$ and the $\Lambda \rightarrow 1$ extrapolation results in $\beta(\Lambda \rightarrow 1) \approx 73.0 \pm 5.0$ (the error bars here are significantly larger as for the extrapolation of the coupling $\alpha$ ). The results from perturbative RG, Sec. III] specifically eqs. (8) and (10), yield $\beta(r)=\mu^{2 r-1} \rho_{0}^{2} \bar{V}^{4} 2 \pi^{2} u^{*}$. This gives $\beta=83.3$.

Similar to Fig. 8 above, we show in Fig. 12 a flow diagram for an exponent very close to $1 / 2, r=0.4985$, so that the levels of the QCP only slightly deviate from those of the SC levels. As discussed above, this deviation is proportional to $\sqrt{1 / 2-r}$, see Fig. [12b. The data shown here are all for subspaces with $(Q=-1, S=0$, $S_{z}=0$ ); the unperturbed energies $E$ of these subspaces are:

- $E=0$ : the levels at $E_{N} \Lambda^{N / 2} \approx 0$, see App. B2

- $E=\epsilon_{2}$ : the levels at $E_{N} \Lambda^{N / 2} \approx 0.8$, see App. B3

- $E=2 \epsilon_{2}$ : the levels at $E_{N} \Lambda^{N / 2} \approx 1.6$, see the example discussed in this section,

- $E=\epsilon_{4}$ : the levels at $E_{N} \Lambda^{N / 2} \approx 1.8$, see App. B4

- $E=3 \epsilon_{2}$ : the levels at $E_{N} \Lambda^{N / 2} \approx 2.4$.

We again find a very good agreement between the perturbative results (crosses) and the NRG data (lines).

Thus we can summarize that our renormalized perturbation theory for the NRG many-body spectrum works well in the vicinity of both $r=0$ and $r=1 / 2$. In principle, from the many-body spectrum (and suitable matrix elements) all other observables like thermodynamic data and dynamic correlation functions can be calculated. We note that the convergence radius of the epsilon-expansion for the levels seems to be smaller than that of the direct epsilon-expansion for certain observables like critical exponents and impurity susceptibility and entropy, see Ref. 13 .

\section{CONCLUSIONS}

Using the quantum phase transitions in the soft-gap Anderson model as an example, we have demonstrated that epsilon-expansion techniques can be used to determine complete many-body spectra at quantum critical points. To this end, we have connected information from standard perturbative RG, which yields information on critical dimensions and parametrically small couplings, and from NRG for the many-body spectra of free-fermion 

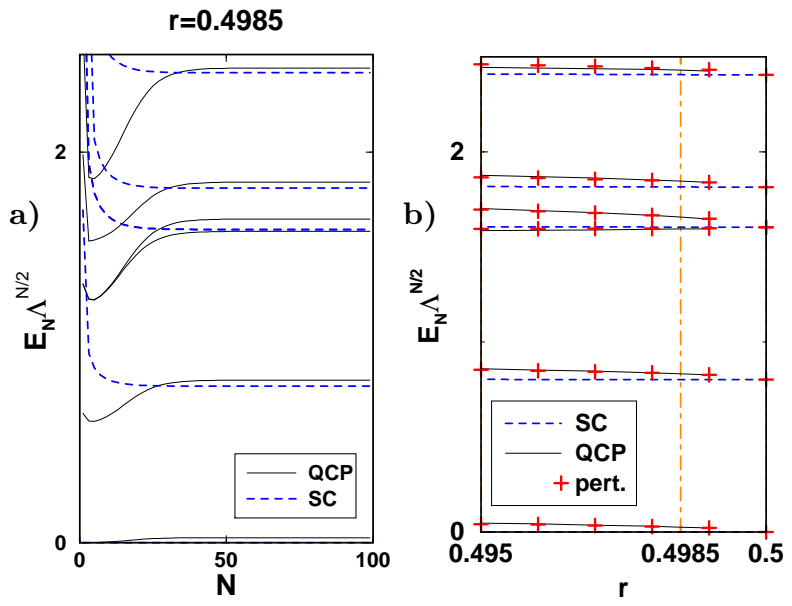

FIG. 12: a) Flow diagram of the lowest lying energy levels for $r=0.4985$; dashed lines: flow to the SC fixed point; solid lines: flow to the quantum critical fixed point. b) The deviation of the QCP levels from the SC levels is proportional to $\sqrt{1 / 2-r}$. This deviation together with the splitting of the energy levels can be explained by the perturbative calculation (crosses) as described in the text.

fixed points. Together, these can be used to perform renormalized perturbation theory for many-body spectra of interacting intermediate-coupling fixed points. For the soft-gap Anderson model, which features two lowercritical dimensions at $r=0$ and $r=1 / 2$, correspondingly two different approaches can be utilized to capture the same critical fixed point: Near $r=0$ a Kondo term has to be added to a free-fermion chain with a decoupled impurity, whereas near $r=1 / 2$ an on-site repulsion is used as a perturbation to the non-interacting Anderson (or resonant-level) model. These perturbations lift the large degeneracies present in the non-interacting spectra, and accurately reproduce the critical spectra determined in NRG calculations at criticality.

Vice versa, our method will be useful in situations where the effective low-energy theory for the critical point is not known: a careful analysis of the many-body spectrum near critical dimensions yields information about the scaling dimension and structure of the relevant operators.

For instance, a plot similar to Fig. 3] can be calculated for the spin-boson model, using the numerical renormalization group method as in Ref. 5. Preliminary results (not shown here) indicate that the many-particle levels of the QCP approach the levels of the delocalized (localized) fixed point in the limit $s \rightarrow 0(s \rightarrow 1)$, with $s$ the exponent of the bath spectral function $J(\omega) \propto \omega^{s}$.

We envision applications of our ideas to more complex impurity models, e.g., with two orbitals or two coupled spins, as well as to non-equilibrium situations treated using $\mathrm{NRG}^{24}$.

\section{Acknowledgments}

We thank S. Kehrein, Th. Pruschke, and A. Rosch for discussions and S. Florens, L. Fritz, M. Kirćan, and N. Tong for collaborations on related work. This research was supported by the DFG through SFB 484 (HJL, RB) and the Center for Functional Nanostructures Karlsruhe (MV). MV also acknowledges support from the Helmholtz Virtual Quantum Phase Transitions Institute in Karlsruhe.

\section{APPENDIX A: DETAILS OF THE PERTURBATIVE ANALYSIS AROUND THE LOCAL MOMENT FIXED POINT}

In this Appendix, we want to give more details for the derivation of the matrix $W_{i j}$ eq. (27) which determines the splitting of the fourfold degeneracy of the subspace ( $Q=0, S=1 / 2, S_{z}=1 / 2$ ) of the LM fixed point at energy $\epsilon_{1}+\epsilon_{3}$. We focus on the matrix element $W_{12}$ :

$$
W_{12}=\left\langle\psi_{1}\left|H_{N}^{\prime}\right| \psi_{2}\right\rangle=\alpha(r) f(N)\left\langle\psi_{1}\left|\vec{S}_{\mathrm{imp}} \cdot \vec{s}_{0}\right| \psi_{2}\right\rangle
$$

The strategy for the calculations can be extended to the other matrix elements and the other subspaces, for which we add the perturbative results at the end of this appendix without derivation. The operator $\vec{S}_{\mathrm{imp}} \cdot \vec{s}_{0}$ is decomposed in four parts:

$$
\begin{aligned}
\vec{S}_{\mathrm{imp}} \cdot \vec{s}_{0}= & \frac{1}{2} S_{\mathrm{imp}}^{+} c_{0 \downarrow}^{\dagger} c_{0 \uparrow}+\frac{1}{2} S_{\mathrm{imp}}^{-} c_{0 \uparrow}^{\dagger} c_{0 \downarrow} \\
& +\frac{1}{2} S_{\mathrm{imp}}^{z}\left(c_{0 \uparrow}^{\dagger} c_{0 \uparrow}-c_{0 \downarrow}^{\dagger} c_{0 \downarrow}\right),
\end{aligned}
$$

so that $W_{12}$ can be written as

$$
W_{12}=\alpha(r) f(N) \frac{1}{2}[\mathrm{I}+\mathrm{II}+\mathrm{III}-\mathrm{IV}],
$$

with

$$
\mathrm{I}=\left\langle\psi_{1}\left|S_{\mathrm{imp}}^{+} c_{0 \downarrow}^{\dagger} c_{0 \uparrow}\right| \psi_{2}\right\rangle,
$$

and the other terms accordingly. With the definitions of $\left|\psi_{1}\right\rangle$ and $\left|\psi_{2}\right\rangle$ of eq. (24) we have:

$$
\begin{aligned}
\mathrm{I}= & \frac{1}{\sqrt{2}}\left\langle\psi_{0}\right|\left(\xi_{-3 \uparrow}^{\dagger} \xi_{1 \uparrow}+\xi_{-3 \downarrow}^{\dagger} \xi_{1 \downarrow}\right) f_{\uparrow} S_{\mathrm{imp}}^{+} c_{0 \downarrow}^{\dagger} c_{0 \uparrow} \\
& \times\left[\frac{1}{\sqrt{6}} f_{\uparrow}^{\dagger}\left(\xi_{1 \uparrow}^{\dagger} \xi_{-3 \uparrow}-\xi_{1 \downarrow}^{\dagger} \xi_{-3 \downarrow}\right)+\frac{2}{\sqrt{6}} f_{\downarrow}^{\dagger} \xi_{1 \uparrow}^{\dagger} \xi_{-3 \downarrow}\right]\left|\psi_{0}\right\rangle .
\end{aligned}
$$

With $S_{\text {imp }}^{+}=f_{\uparrow}^{\dagger} f_{\downarrow}$ we immediately see that the terms containing $f_{\uparrow} S_{\text {imp }}^{+} f_{\uparrow}^{\dagger}$ drop out. The remaining impurity operators, $f_{\uparrow} S_{\mathrm{imp}}^{+} f_{\downarrow}^{\dagger}$, give unity when acting on $\left|\psi_{0}\right\rangle$ so one arrives at

$$
\mathrm{I}=\frac{1}{\sqrt{3}}[\mathrm{Ia}+\mathrm{Ib}]
$$


with

$$
\begin{aligned}
& \mathrm{Ia}=\left\langle\psi_{0}\left|\xi_{-3 \uparrow}^{\dagger} \xi_{1 \uparrow} c_{0 \downarrow}^{\dagger} c_{0 \uparrow} \xi_{1 \uparrow}^{\dagger} \xi_{-3 \downarrow}\right| \psi_{0}\right\rangle \\
& \mathrm{Ib}=\left\langle\psi_{0}\left|\xi_{-3 \downarrow}^{\dagger} \xi_{1 \downarrow} c_{0 \downarrow}^{\dagger} c_{0 \uparrow} \xi_{1 \uparrow}^{\dagger} \xi_{-3 \downarrow}\right| \psi_{0}\right\rangle .
\end{aligned}
$$

To analyze Ia and Ib, the operators $c_{0 \sigma}^{(\dagger)}$ have to be expressed in terms of the operators $\xi_{p \sigma}^{(\dagger)}$ :

$$
c_{0 \sigma}=\sum_{p^{\prime}} \alpha_{0 p^{\prime}} \xi_{p^{\prime} \sigma}, \quad c_{0 \sigma}^{\dagger}=\sum_{p} \alpha_{0 p}^{*} \xi_{p \sigma}^{\dagger},
$$

with the sums over $p$ and $p^{\prime}$ defined in eq. (20). This gives

$$
\mathrm{Ia}=\sum_{p p^{\prime}} \alpha_{0 p}^{*} \alpha_{0 p^{\prime}}\left\langle\psi_{0}\left|\xi_{-3 \uparrow}^{\dagger} \xi_{1 \uparrow} \xi_{p \downarrow}^{\dagger} \xi_{p^{\prime} \uparrow} \xi_{1 \uparrow}^{\dagger} \xi_{-3 \downarrow}\right| \psi_{0}\right\rangle .
$$

The only non-zero matrix elements of eq. A9 are for $p=p^{\prime}=-3$ :

$$
\begin{aligned}
\mathrm{Ia} & =\alpha_{0-3}^{*} \alpha_{0-3}\left\langle\psi_{0}\left|\xi_{-3 \uparrow}^{\dagger} \xi_{1 \uparrow} \xi_{-3 \downarrow}^{\dagger} \xi_{-3 \uparrow} \xi_{1 \uparrow}^{\dagger} \xi_{-3 \downarrow}\right| \psi_{0}\right\rangle \\
& =-\left|\alpha_{0-3}\right|^{2}
\end{aligned}
$$

Similarly, the term Ib gives

$$
\begin{aligned}
\mathrm{Ib} & =\sum_{p p^{\prime}} \alpha_{0 p}^{*} \alpha_{0 p^{\prime}}\left\langle\psi_{0}\left|\xi_{-3 \downarrow}^{\dagger} \xi_{1 \downarrow} \xi_{p \downarrow}^{\dagger} \xi_{p^{\prime} \uparrow} \xi_{1 \uparrow}^{\dagger} \xi_{-3 \downarrow}\right| \psi_{0}\right\rangle \\
& =\left|\alpha_{01}\right|^{2}
\end{aligned}
$$

so that in total:

$$
I=\frac{1}{\sqrt{3}}\left[-\left|\alpha_{0-3}\right|^{2}+\left|\alpha_{01}\right|^{2}\right]
$$

The next term II $=\left\langle\psi_{1}\left|S_{\mathrm{imp}}^{-} c_{0 \uparrow}^{\dagger} c_{0 \downarrow}\right| \psi_{2}\right\rangle$ gives zero due to the combination of impurity operators: $f_{\uparrow} f_{\downarrow}^{\dagger} f_{\uparrow} \ldots$ with $f_{\uparrow}$ from $\left\langle\psi_{1}\right|$ and $f_{\downarrow}^{\dagger} f_{\uparrow}=S_{\text {imp }}^{-}$.

The third term III $=\left\langle\psi_{1}\left|S_{\mathrm{imp}}^{z} c_{0 \uparrow}^{\dagger} c_{0 \uparrow}\right| \psi_{2}\right\rangle$ gives

$$
\begin{aligned}
\mathrm{III}= & \frac{1}{\sqrt{12}}\left\langle\psi_{0}\right|\left(\xi_{-3 \uparrow}^{\dagger} \xi_{1 \uparrow}+\xi_{-3 \downarrow}^{\dagger} \xi_{1 \downarrow}\right) f_{\uparrow} S_{\mathrm{imp}}^{z} c_{0 \uparrow}^{\dagger} c_{0 \uparrow} f_{\uparrow}^{\dagger} \\
& \times\left(\xi_{1 \uparrow}^{\dagger} \xi_{-3 \uparrow}-\xi_{1 \downarrow}^{\dagger} \xi_{-3 \downarrow}\right)\left|\psi_{0}\right\rangle,
\end{aligned}
$$

where the term with $\frac{2}{\sqrt{6}} f_{\downarrow}^{\dagger} \xi_{1 \uparrow}^{\dagger} \xi_{-3 \downarrow}$ from $\left|\psi_{2}\right\rangle$ has already been dropped. So we are left with four terms

$$
\mathrm{III}=\frac{1}{\sqrt{12}}[\mathrm{IIIa}-\mathrm{IIIb}+\mathrm{IIIc}-\text { IIId }],
$$

with

$$
\begin{aligned}
& \text { IIIa }=\left\langle\psi_{0}\left|\xi_{-3 \uparrow}^{\dagger} \xi_{1 \uparrow} f_{\uparrow} S_{\text {imp }}^{z} c_{0 \uparrow}^{\dagger} c_{0 \uparrow} f_{\uparrow}^{\dagger} \xi_{1 \uparrow}^{\dagger} \xi_{-3 \uparrow}\right| \psi_{0}\right\rangle, \\
& \text { IIIb }=\left\langle\psi_{0}\left|\xi_{-3 \uparrow}^{\dagger} \xi_{1 \uparrow} f_{\uparrow} S_{\text {imp }}^{z} c_{0 \uparrow}^{\dagger} c_{0 \uparrow} f_{\uparrow}^{\dagger} \xi_{1 \downarrow}^{\dagger} \xi_{-3 \downarrow}\right| \psi_{0}\right\rangle, \\
& \text { IIIc }=\left\langle\psi_{0}\left|\xi_{-3 \downarrow}^{\dagger} \xi_{1 \downarrow} f_{\uparrow} S_{\text {imp }}^{z} c_{0 \uparrow}^{\dagger} c_{0 \uparrow} f_{\uparrow}^{\dagger} \xi_{1 \uparrow}^{\dagger} \xi_{-3 \uparrow}\right| \psi_{0}\right\rangle, \\
& \text { IIId }=\left\langle\psi_{0}\left|\xi_{-3 \downarrow}^{\dagger} \xi_{1 \downarrow} f_{\uparrow} S_{\text {imp }}^{z} c_{0 \uparrow}^{\dagger} c_{0 \uparrow} f_{\uparrow}^{\dagger} \xi_{1 \downarrow}^{\dagger} \xi_{-3 \downarrow}\right| \psi_{0}\right\rangle .
\end{aligned}
$$

Following similar arguments as above one obtains

$$
\mathrm{IIIa}=\frac{1}{2} \sum_{p}^{\prime}\left|\alpha_{0 p}\right|^{2},
$$

where the $p$ in $\sum_{p}{ }^{\prime}$ takes the values

$$
p=1,-1,-5,-7, \ldots-N,
$$

then

$$
\mathrm{IIIb}=\mathrm{IIIc}=0,
$$

and

$$
\operatorname{IIId}=\frac{1}{2} \sum_{p}^{\prime \prime}\left|\alpha_{0 p}\right|^{2}
$$

where the $p$ in $\sum_{p}{ }^{\prime \prime}$ takes the values

$$
p=-1,-3,-5,-7, \ldots-N \text {. }
$$

This gives for the third term

$$
\begin{aligned}
\text { III } & =\frac{1}{\sqrt{12}}[\text { IIIa }- \text { IIId }] \\
& =\frac{1}{4 \sqrt{3}}\left[\sum_{p}^{\prime}\left|\alpha_{0 p}\right|^{2}-\sum_{p}^{\prime \prime}\left|\alpha_{0 p}\right|^{2}\right] \\
& =\frac{1}{4 \sqrt{3}}\left[\left|\alpha_{01}\right|^{2}-\left|\alpha_{0-3}\right|^{2}\right] .
\end{aligned}
$$

The calculation of IV proceeds very similarly to III and one obtains

$$
\mathrm{III}=-\mathrm{IV},
$$

so that we finally arrive at

$$
\begin{aligned}
W_{12} & =\alpha(r) f(N) \frac{1}{2}\left(\left|\alpha_{01}\right|^{2}-\left|\alpha_{0-3}\right|^{2}\right)\left[\frac{1}{\sqrt{3}}+0+2 \frac{1}{4 \sqrt{3}}\right] \\
& =\alpha(r) f(N) \frac{1}{4} \sqrt{3}\left(\left|\alpha_{01}\right|^{2}-\left|\alpha_{0-3}\right|^{2}\right) .
\end{aligned}
$$

We performed a similar analysis for a couple of other subspaces. Here we list the results from the perturbative analysis for three more subspaces together with the corresponding basis states.

$$
\text { 1. } Q=0, S=1 / 2, S_{z}=1 / 2, E=2 \epsilon_{1}
$$

This subspace has the same quantum numbers $Q, S$ and $S_{z}$ as the one discussed above, so that the details of the calculation are very similar. The differences originate from the position of particles and holes in the singleparticle spectrum of Fig. [6] This reduces the dimensionality of the subspace from four to two. 
The corresponding basis can be written as

$\left|\psi_{1}\right\rangle=\frac{1}{\sqrt{2}} f_{\uparrow}^{\dagger}\left(\xi_{1 \uparrow}^{\dagger} \xi_{-1 \uparrow}+\xi_{1 \downarrow}^{\dagger} \xi_{-1 \downarrow}\right)\left|\psi_{0}\right\rangle$,
$\left|\psi_{2}\right\rangle=\left[\frac{1}{\sqrt{6}} f_{\uparrow}^{\dagger}\left(\xi_{1 \uparrow}^{\dagger} \xi_{-1 \uparrow}-\xi_{1 \downarrow}^{\dagger} \xi_{-1 \downarrow}\right)+\frac{2}{\sqrt{6}} f_{\downarrow}^{\dagger} \xi_{1 \uparrow}^{\dagger} \xi_{-1 \downarrow}\right]\left|\psi_{0}\right\rangle$.

The first-order corrections are given by the $2 \times 2$ matrix

$$
\left\{W_{i j}\right\}=\alpha(r) f(N)\left[\begin{array}{cc}
0 & \frac{\sqrt{3}}{4} \gamma \\
\frac{\sqrt{3}}{4} \gamma & -\frac{1}{2} \beta
\end{array}\right],
$$

with $\gamma=\left|\alpha_{01}\right|^{2}-\left|\alpha_{0-1}\right|^{2}$ and $\beta=\left|\alpha_{01}\right|^{2}+\left|\alpha_{0-1}\right|^{2}$. Due to the particle-hole symmetry of the conduction band we have $\left|\alpha_{01}\right|=\left|\alpha_{0-1}\right|$; therefore, the off-diagonal matrix elements vanish and the effect of the perturbation is simply a negative energy-shift only for the state $\left|\psi_{2}\right\rangle$ :

$$
\left\{W_{i j}\right\}=\alpha(r) f(N)\left[\begin{array}{cc}
0 & 0 \\
0 & -\left|\alpha_{01}\right|^{2}
\end{array}\right] .
$$

This effect can be seen in the energy splitting of the first two low-lying excitations in Fig. 8

$$
\text { 2. } Q=-1, S=0, E=-\epsilon_{-1}
$$

There is only one configuration for this combination of quantum numbers and excitation energy:

$$
|\psi\rangle=\frac{1}{\sqrt{2}}\left(f_{\uparrow}^{\dagger} \xi_{-1 \uparrow}+f_{\downarrow}^{\dagger} \xi_{-1 \downarrow}\right)\left|\psi_{0}\right\rangle .
$$

The first-order perturbation keeps the state in this onedimensional subspace and the energy correction is given by

$$
\Delta E=\left\langle\psi\left|H_{N}^{\prime}\right| \psi\right\rangle=-\frac{3}{4} \alpha(r) f(N)\left|\alpha_{0-1}\right|^{2} .
$$

$$
\text { 3. } Q=-1, S=0, E=-\epsilon_{-3}
$$

The difference to the previous case is the position of the hole in the single-particle spectrum. The state is now given by

$$
|\psi\rangle=\frac{1}{\sqrt{2}}\left(f_{\uparrow}^{\dagger} \xi_{-3 \uparrow}+f_{\downarrow}^{\dagger} \xi_{-3 \downarrow}\right)\left|\psi_{0}\right\rangle,
$$

with the energy correction

$$
\Delta E=\left\langle\psi\left|H_{N}^{\prime}\right| \psi\right\rangle=-\frac{3}{4} \alpha(r) f(N)\left|\alpha_{0-3}\right|^{2} .
$$

\section{APPENDIX B: DETAILS OF THE PERTURBATIVE ANALYSIS AROUND THE STRONG COUPLING FIXED POINT}

The main difference in the calculation of the matrix elements $\left\{W_{i j}\right\}$ for this case is due to the structure of the perturbation, see eq. (37). Furthermore the ground state of the SC fixed point is fourfold degenerate and the perturbation partially splits this degeneracy, as discussed in the following.

$$
\text { 1. } Q=0, S=1 / 2, S_{z}=1 / 2, E=0
$$

This is one of the four degenerate ground states at the SC fixed point:

$$
\left|\psi_{1}\right\rangle=\xi_{0 \uparrow}^{\dagger}\left|\psi_{0}\right\rangle,
$$

with $\left|\psi_{0}\right\rangle$ defined in eq. (41).

The perturbative correction is given by

$$
\left\langle\psi_{1}\left|H_{N}^{\prime}\right| \psi_{1}\right\rangle=\frac{1}{2} \beta(r) \bar{f}(N)\left(1-\left|\alpha_{f 0}\right|^{4}\right)
$$

which corresponds to the energy shift of the ground state:

$$
\Delta E_{1}=\frac{1}{2} \beta(r) \bar{f}(N)\left(1-\left|\alpha_{f 0}\right|^{4}\right) .
$$

The coefficients $\alpha_{f l}$ are defined via the relation between the operators $f_{\sigma}^{(\dagger)}$ and $\xi_{l \sigma}^{(\dagger)}$ :

$$
f_{\sigma}=\sum_{l^{\prime}} \alpha_{f l^{\prime}} \xi_{l^{\prime} \sigma}, \quad f_{\sigma}^{\dagger}=\sum_{l} \alpha_{f l}^{*} \xi_{l \sigma}^{\dagger} .
$$

$$
\text { 2. } Q=-1, S=0, E=0
$$

This state is also a ground state in the $U=0$ case:

$$
\left|\psi_{2}\right\rangle=\left|\psi_{0}\right\rangle .
$$

The calculation of the first-order correction for $\left|\psi_{2}\right\rangle$ gives

$$
\left\langle\psi_{2}\left|H_{N}^{\prime}\right| \psi_{2}\right\rangle=\frac{1}{2} \beta(r) \bar{f}(N)\left(1+\left|\alpha_{f 0}\right|^{4}\right) .
$$

This means that the ground state including the effect of the perturbation is given by $\left|\psi_{1}\right\rangle$ in eq. (B1) and the state $\left|\psi_{2}\right\rangle$ appears as an excited state. For a comparison with the energy levels shown in the NRG flow diagrams, where the ground state energy is set to zero in each iteration, we subtract the perturbative correction of the ground state $\left(\Delta E_{1}\right)$ from the energies of all other excited states. Subtracting this energy shift from eq. (B6) gives the net energy correction for the $\left|\psi_{2}\right\rangle$ state:

$$
\Delta E_{2}=\beta(r) \bar{f}(N)\left|\alpha_{f 0}\right|^{4} .
$$




$$
\text { 3. } Q=-1, S=0, E=\epsilon_{2}
$$

The state corresponding to this subspace is given by:

$$
\left|\psi_{3}\right\rangle=\frac{1}{\sqrt{2}}\left(\xi_{0 \uparrow}^{\dagger} \xi_{-2 \uparrow}+\xi_{0 \downarrow}^{\dagger} \xi_{-2 \downarrow}\right)\left|\psi_{0}\right\rangle .
$$

The first-order correction reads

$\left\langle\psi_{3}\left|H_{N}^{\prime}\right| \psi_{3}\right\rangle=\beta(r) \bar{f}(N)\left[\frac{1}{2}\left(1-\left|\alpha_{f 0}\right|^{4}\right)+3\left|\alpha_{f 0}\right|^{2}\left|\alpha_{f-2}\right|^{2}\right]$

Subtracting the energy correction for the ground state results in

$$
\Delta E_{3}=3 \beta(r) \bar{f}(N)\left|\alpha_{f 0}\right|^{2}\left|\alpha_{f-2}\right|^{2} .
$$

$$
\text { 4. } Q=-1, S=0, E=\epsilon_{4}
$$

Similarly for the state

$$
\left|\psi_{4}\right\rangle=\frac{1}{\sqrt{2}}\left(\xi_{0 \uparrow}^{\dagger} \xi_{-4 \uparrow}+\xi_{0 \downarrow}^{\dagger} \xi_{-4 \downarrow}\right)\left|\psi_{0}\right\rangle
$$

the first-order correction is given by
$\left\langle\psi_{4}\left|H_{N}^{\prime}\right| \psi_{4}\right\rangle=\beta(r) \bar{f}(N)\left[\frac{1}{2}\left(1-\left|\alpha_{f 0}\right|^{4}\right)+3\left|\alpha_{f 0}\right|^{2}\left|\alpha_{f-4}\right|^{2}\right]$

and subtracting the energy correction for the ground state results in:
${ }^{1}$ R. Bulla and M. Vojta, in Concepts in Electron Correlations, A.C. Hewson and V. Zlatić (eds.), Kluwer Academic Publishers, Dordrecht (2003), 209; M. Vojta, cond-mat/0412208

2 Sec. 4 in: M. Vojta, Rep. Prog. Phys. 66, 2069 (2003).

3 I. Affleck, J. Phys. Soc. Jpn. 74, 59 (2005).

4 A. J. Leggett, S. Chakravarty, A. T. Dorsey, M. P. A. Fisher, A. Garg, and W. Zwerger, Rev. Mod. Phys. 59, 1 (1987).

${ }^{5}$ R. Bulla, N. Tong, and M. Vojta, Phys. Rev. Lett. 91, 170601 (2003).

6 M. Vojta and M. Kirćan, Phys. Rev. Lett. 90, 157203 (2003); M. Kirćan and M. Vojta, Phys. Rev. B 69, 174421 (2004).

7 L. De Leo and M. Fabrizio, Phys. Rev. Lett. 94, 236401 (2005).

8 N. J. Craig, J. M. Taylor, E. A. Lester, C. M. Marcus, M. P. Hanson, and A. C. Gossard, Science 304, 565 (2004).

9 K. Le Hur, Phys. Rev. Lett. 92, 196804 (2004); M.-R. Li and K. Le Hur, Phys. Rev. Lett. 93, 176802 (2004).

10 D. V. Khveshchenko, Phys. Rev. B 69, 153311 (2004).

11 Q. Si, S. Rabello, K. Ingersent, and J. L. Smith, Nature 413, 804 (2001); Phys. Rev. B 68, 115103 (2003).

12 D. Withoff and E. Fradkin, Phys. Rev. Lett. 64, 1835
(1990)

13 M. Vojta and L. Fritz, Phys. Rev. B 70, 094502 (2004); L. Fritz and M. Vojta, Phys. Rev. B 70, 214427 (2004).

14 D. E. Logan and M. T. Glossop, J. Phys.: Condens. Matter 12, 985 (2000).

15 M.T. Glossop and D.E. Logan, J. Phys.: Condens. Matter 15, 7519 (2003).

16 K. G. Wilson, Rev. Mod. Phys. 47, 773 (1975).

17 R. Bulla, A. C. Hewson, and G.-M. Zhang, Phys. Rev. B 56, 11721 (1997).

18 A. C. Hewson, The Kondo Problem to Heavy Fermions (Cambridge Univ. Press, Cambridge 1993).

19 C. Gonzalez-Buxton and K. Ingersent, Phys. Rev. B 57, 14254 (1998).

20 R. Bulla, T. Pruschke, and A. C. Hewson, J. Phys.: Condens. Matter 9, 10463 (1997); R. Bulla, M. T. Glossop, D. E. Logan, and T. Pruschke, ibid 12, 4899 (2000).

21 M. Vojta and R. Bulla, Phys. Rev. B 65, 014511 (2002).

22 K. Chen and C. Jayaprakash, J. Phys.: Condens. Matter 7, L491 (1995).

23 H. R. Krishna-murthy, J. W. Wilkins, and K. G. Wilson, Phys. Rev. B 21, 1003 (1980); ibid. 21, 1044 (1980).

24 F. B. Anders and A. Schiller, cond-mat/0505553 